\title{
Dosimetric benefit to organs at risk following margin reductions in nasopharyngeal carcinoma treated with intensity-modulated radiation therapy
}

\author{
Yan-Ping Mao ${ }^{1 \dagger}$, Wen-Jing Yin ${ }^{2 \dagger}$, Rui Guo ${ }^{1}$, Guang-Shun Zhang ${ }^{1}$, Jian-Lan Fang ${ }^{1}$, Feng Chi ${ }^{1}$, Zhen-Yu Qi ${ }^{1}$, \\ Meng-Zhong Liu', Jun Ma ${ }^{1}$ and Ying Sun ${ }^{1 *}$
}

\begin{abstract}
Introduction: It is important to decrease the radiation exposure of normal tissue in intensity-modulated radiation therapy (IMRT). Minimizing planning target volume (PTV) margins with more precise target localization techniques can achieve this goal. This study aimed to quantify the extent to which organs at risk (OARs) are spared when using reduced margins in the treatment of nasopharyngeal carcinoma (NPC).

Methods: Two IMRT plans were regenerated for 40 patients with NPC based on two PTV margins, which were reduced or unchanged following cone beam computed tomography online correction. The reduced-margin plan was optimized based on maximal dose reduction to OARs without compromising target coverage. Dosimetric comparisons were evaluated in terms of target coverage and OAR sparing.

Results: Improvements in target coverage occurred with margin reduction, and significant improvements in dosimetric parameters were observed for all OARs $(P<0.05)$ except for the right optic nerve, chiasm, and lens. Doses to OARs decreased at a rate of $1.5 \%$ to $7.7 \%$. Sparing of the left parotid and right parotid, where the mean dose $\left(D_{\text {mean }}\right)$ decreased at a rate of $7.1 \%$ and $7.7 \%$, respectively, was greater than the sparing of other OARs.
\end{abstract}

Conclusions: Significant improvements in OAR sparing were observed with margin reduction, in addition to improvement in target coverage. The parotids benefited most from the online imaging-guided approach.

Keywords: Margin, Dosimetry, Organs at risk, Cone beam computer tomography, Nasopharyngeal carcinoma

\section{Background}

Nasopharyngeal carcinoma (NPC) is endemic in particular regions such as Southeast Asia. The annual incidence varies from 15 to 50 per 100,000 people in South China [1]. Radiation therapy is the primary approach for treating locoregionally confined NPC.

Intensity-modulated radiation therapy (IMRT) provides excellent locoregional control and sparing of organs at risk (OARs) in NPC [2-4] and has gradually replaced twodimensional conventional radiotherapy as the first-line

\footnotetext{
*Correspondence: sunying2@mail.sysu.edu.cn

${ }^{\dagger}$ Equal contributors

'Department of Radiation Oncology, Sun Yat-sen University Cancer Center; State Key Laboratory of Oncology in South China; Collaborative Innovation Center for Cancer Medicine, Guangzhou, Guangdong 510060, Peoples Republic of China Full list of author information is available at the end of the article
}

radiotherapy technique. Although the sparing of OARs has improved significantly with IMRT, late toxicities such as grades 2 to 4 xerostomia and sensorineural hearing loss still occur with incidences of $39.3 \%$ and $37.0 \%$, respectively $[4,5]$. Therefore, improvements in OAR sparing and reductions in radiation toxicity remain to be important issues. Minimizing planning target volume (PTV) margins with more precise target localization techniques decreases the dose delivered to OARs [6-8]. However, little data have quantified the extent to which OARs are spared by reducing margins in NPC radiotherapy up to date.

Safety margins can be reduced with many imagingguided radiation therapy technologies. By providing better resolution and three-dimensional (3D) images, conebeam computed tomography $(\mathrm{CBCT})$ allows for a more 
accurate verification of the pretreatment position. Furthermore, the combination of $\mathrm{CBCT}$ imaging equipment, treatment couch, and automatic software for online correction allows for real-time monitoring and accurate online corrections [6-10]. Current literature has reported that $\mathrm{CBCT}$ online corrections can shrink safety margins in head and neck cancer by approximately $50 \%[11,12]$. Quantification of the potential dosimetric benefit from margin reductions with $\mathrm{CBCT}$ online correction in NPC would be helpful to evaluate the value of CBCT.

Therefore, we generated two radiotherapy plans with pre-correction and post-correction PTV margins obtained through $\mathrm{CBCT}$ online correction. We then quantified the extent of OAR sparing with margin reductions without compromising PTV coverage. Additionally, we examined whether the dosimetric benefits observed from margin reductions using CBCT were associated with tumor stage and volume.

\section{Patients and Methods}

\section{Patient characteristics}

Between October 2010 and October 2011, 40 consecutive patients with newly diagnosed, untreated, and nondisseminated NPC were retrospectively included in the study. Approval for retrospective analysis of the patient data was obtained from the ethics committee of Sun Yat-sen University Cancer Center. Written consent was waived, and oral consent from the patients was obtained via telephone and documented by telephone recording. The use of oral consent was approved by the Institutional Review Board.

All patients underwent a pre-treatment evaluation, which included a complete medical history, physical and neurological examinations, hematology and biochemistry profiles, magnetic resonance imaging (MRI) scans of the neck and nasopharynx, chest radiography, and abdominal ultrasonography. Patients with N2 or N3 lesions underwent emission computed tomography (ECT) or positron emission tomography-computed topography (PET-CT). Medical records and images were analyzed retrospectively, and all cases were staged according to the 7th edition American Joint Committee on Cancer (AJCC) staging system. Clinical characteristics are listed in Table 1.

\section{Treatment}

All patients were fixed with a 5-point thermoplastic mask (Civco Medical Solutions, Kolona, IA, USA), which may also position the patients' shoulders. Target volumes were delineated according to the International Commission on Radiation Units and Measurements (ICRU) reports 50 and 62. The PTVs and planning organs at risk volume (PRVs) were generated by the addition of a 3-mm margin to both the delineated target volume and corresponding structures (such as the spinal cord, brainstem, and optic
Table 1 Clinical characteristics of the $\mathbf{4 0}$ patients with nasopharyngeal carcinoma (NPC)

\begin{tabular}{|c|c|}
\hline Characteristic & No. of patients (\%) \\
\hline \multicolumn{2}{|l|}{ Age (years) } \\
\hline Median & 46 \\
\hline Range & $21-63$ \\
\hline \multicolumn{2}{|l|}{ Sex } \\
\hline Male & $32(80.0)$ \\
\hline Female & $8(20.0)$ \\
\hline \multicolumn{2}{|l|}{ Histology } \\
\hline WHO I & $0(0)$ \\
\hline WHO $\|/\| I$ & $40(100.0)$ \\
\hline \multicolumn{2}{|l|}{ T category $^{a}$} \\
\hline $\mathrm{T} 1$ & $7(17.5)$ \\
\hline $\mathrm{T} 2$ & $9(22.5)$ \\
\hline T3 & $15(37.5)$ \\
\hline T4 & $9(22.5)$ \\
\hline \multicolumn{2}{|l|}{$N$ category $^{a}$} \\
\hline No & $10(25.0)$ \\
\hline N1 & $23(57.5)$ \\
\hline N2 & $6(15.0)$ \\
\hline N3 & $1(2.5)$ \\
\hline \multicolumn{2}{|l|}{ Clinical stage ${ }^{a}$} \\
\hline I & $5(12.5)$ \\
\hline$\|$ & $10(25.0)$ \\
\hline III & $15(37.5)$ \\
\hline IV & $10(25.0)$ \\
\hline \multicolumn{2}{|l|}{ Chemotherapy } \\
\hline No & $8(20.0)$ \\
\hline Yes & $32(80.0)$ \\
\hline
\end{tabular}

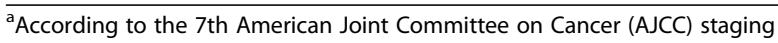
system. WHO, World Health Organization.

nerve pathway). The prescribed dose was 70 Gy to the PTV of the gross tumor volume of the primary site (GTV-P), 64-66 Gy to the PTV of the nodal gross tumor volume (GTV-N), 60 Gy to the PTV of the clinical target volume1 (CTV-1; high-risk regions), and 56 Gy to the PTV of the CTV-2 (low-risk regions) and the CTV-N (nodal regions in the neck) in 33 fractions. The PTVs of GTV, CTV1, and CTV2 were named PTV_7000, PTV_6000, and PTV_5600, respectively. All patients were treated with 1 fraction daily, 5 days a week. Neoadjuvant and adjuvant chemotherapy and concomitant chemotherapy with a platinum-based protocol were recommended for patients with stage III to IVB NPC.

\section{PTV margins}

To study the dosimetric impact of margin reductions on the IMRT plan, two additional PTVs with pre-correction 
and post-correction PTV margins were generated for each patient. We obtained the pre-correction and postcorrection PTV margins through our preliminary study. The process was as follows.

First, after conventional positioning by aligning the inroom lasers with the marks drawn on the masks, we obtained the first CBCT images. Second, the acquired CBCT images were registered to the planning $\mathrm{CT}$ scan by using automatic bone matching (Elekta XVI software, Elekta, Crawley, UK) to obtain the translational errors of target on the medial-lateral (ML), superior-inferior (SI), and anterior-posterior (AP) directions. If a translational error was greater than $2 \mathrm{~mm}$ in any direction, setup corrections were made by adjusting the patient's position through automatically shifting the treatment couch in all ML, SI, and AP directions. Third, after setup correction, a second $\mathrm{CBCT}$ scan was performed and registered to the planning $\mathrm{CT}$ scan, thereby obtaining the residual setup error. Finally, the fraction radiation therapy was performed. The difference between the pre-correction CBCT image and planning $\mathrm{CT}$ was pre-correction setup error, whereas the difference between the post-correction CBCT image and planning CT image was post-correction setup error. We followed the geometric margin formula developed by van Herk et al. [13] to calculate the pre-correction and postcorrection PTV margins with pre-correction and postcorrection errors, respectively.

The pre-correction and post-correction systematic setup uncertainty, random setup uncertainty, and PTV margins, obtained during preliminary research in our center, are shown in Table 2. Post-correction margins were clearly smaller than the pre-correction margins in the 3D direction, and the margins decreased by $48 \%-63 \%$ after online correction in the three directions.

\section{Plan re-optimization}

Optimization was performed by using the Monaco treatment planning system (version 3.1, Elekta Medical Systems, Crawley, UK) and doses were calculated with the Monte Carlo algorithm [14]. Both plans with pre-correction and post-correction margins were generated for an Elekta Synergy linear accelerator (Elekta, Crawley, UK) using

Table 2 Pre-correction and post-correction systematic setup uncertainty, random setup uncertainty, and planning target volume (PTV) margins in three-dimensional directions

\begin{tabular}{lccccccc}
\hline Variable & \multicolumn{3}{c}{ Pre-correction } & & & \multicolumn{3}{c}{ Post-correction } \\
\cline { 2 - 3 } & ML & SI & AP & & ML & SI & AP \\
\hline$\Sigma^{\mathrm{a}}(\mathrm{mm})$ & 1.4 & 1.0 & 1.0 & & 0.4 & 0.5 & 0.4 \\
$\sigma^{\mathrm{b}}(\mathrm{mm})$ & 0.8 & 0.8 & 0.7 & & 0.6 & 0.7 & 0.7 \\
PTV $(\mathrm{mm})$ & 4.0 & 3.1 & 3.1 & & 1.5 & 1.7 & 1.6 \\
\hline
\end{tabular}

${ }^{\mathrm{a}}$ Systematic setup uncertainty. ${ }^{\mathrm{b}}$ Random setup uncertainty. $\mathrm{ML}$, medial-lateral; $\mathrm{SI}$, superior-inferior; AP, anterior-posterior.
6-MV photons. A standard constraint set referring to RTOG0615 was used for optimization and evaluation. The aim was to achieve $95 \%$ of any PTV at or above the prescription dose, $99 \%$ of any PTV at or above $93 \%$ of the PTV dose, no more than $20 \%$ of the PTV_7000 at or above $77 \mathrm{~Gy}$ (that is, $110 \%$ of the PTV_7000 dose), and no more than $5 \%$ of any PTV_7000 at or above $80.5 \mathrm{~Gy}$ (that is, $115 \%$ of the PTV_7000 dose). For OARs, the most important objective was to keep maximum doses to the $1 \%$ of the PRV of the spinal cord (SpinalCord_PRV) below 50 Gy and to the $1 \%$ of the PRV of the brain stem (BrainStem_PRV) below 60 Gy. The second priority was to ensure that $50 \%$ of the parotid glands received a dose < 30 Gy (to be achieved in at least one gland). All targets were treated simultaneously by using the simultaneous integrated boost (SIB) technique.

\section{Plan comparison}

A quantitative comparison of plans was performed by using the standard dose-volume histogram (DVH). The DVH parameters for target comparisons refer to ICRU 83. In both plans, the median dose, $\mathrm{D}_{2 \%}, \mathrm{D}_{95 \%}$, and $\mathrm{D}_{98 \%}$ (doses that cover 2\%, 95\%, and 98\% of the PTV, respectively) were recorded for each PTV. Values of $\mathrm{D}_{98 \%}$ and $\mathrm{D}_{2} \%$ were defined as metrics for minimum and maximum doses, respectively. A further measurement of dose homogeneity was expressed by the homogeneity index (HI), which was $\left(\mathrm{D}_{2 \%}-\mathrm{D}_{98 \%}\right) / \mathrm{D}_{50 \%}$, and a high $\mathrm{HI}$ indicates poor homogeneity.

For OARs, the analysis included the maximum dose, the mean dose, and a set of appropriate $V_{X}$ (percentage volume receiving less/more than $\mathrm{X} G y$ ) and $\mathrm{D}_{\mathrm{Y}}$ (dose received by Y volume) values.

\section{Statistical analysis}

All analyses were performed using SPSS software, version 16.0 (SPSS, Chicago, IL, USA). Paired $t$ tests were used to compare the dosimetry of plans with pre-correction margins and those with post-correction margins. Pearson's correlation analysis was used to evaluate associations between differences in DVH parameters for both PTV scenarios and tumor volume, and a one-way analysis of variance (ANOVA) was used to analyze differences in dosimetric improvements with various tumor stages. Two-tailed $P$ values of $<0.05$ were considered significant.

\section{Results}

\section{Target coverage}

The volumes of PTV with post-correction margins were greater than those of PTV with pre-correction margins (Table 3). Irrespective of margin reductions, all plans met the planning goals for target coverage (Table 4). Dosimetric differences in targets that resulted from margin reductions are summarized in Table 5. In general, 
Table 3 The tumor volume for targets in 40 NPC patients

\begin{tabular}{llcrr}
\hline Target & & Mean $(\mathrm{mL})$ & SD $(\mathbf{m L})$ & \multicolumn{1}{c}{ Range $(\mathbf{m L})$} \\
\hline GTV & 35.44 & 29.02 & $1.17-119.70$ \\
CTV1 & 112.04 & 50.42 & $21.69-239.26$ \\
CTV2 & 493.20 & 134.24 & $75.24-792.59$ \\
PTV_7000 & Pre-correction & 68.20 & 42.78 & $4.51-186.32$ \\
& Post-correction & 54.65 & 36.02 & $3.09-161.97$ \\
PTV_6000 & Pre-correction & 166.75 & 66.79 & $40.70-333.05$ \\
& Post-correction & 147.16 & 61.98 & $33.99-299.74$ \\
PTV_5600 & Pre-correction & 745.13 & 155.04 & $364.14-1,108.09$ \\
& Post-correction & 650.69 & 141.54 & $311.18-988.26$ \\
\hline
\end{tabular}

GTV, gross tumor volume; CTV, clinical target volume; PTV_7000, the PTV of gross tumor volume; PTV_6000, the PTV of high-risk regions; PTV_5600, the PTV of low-risk regions; SD, standard deviation. significant improvements in target coverage were observed following margin reductions. Regarding $\mathrm{HI}$ and $\mathrm{D}_{2 \%}$, plans with post-correction margins showed larger values compared with plans with pre-correction margins at all dose levels $(P<0.05)$. The $\mathrm{D}_{98 \%}$ of PTV_7000 was larger for plans with post-correction margins than that for plans with pre-correction margins $(P<0.05)$, but there were no significant differences in PTV_6000 and PTV_5600 ( $P=0.805$ and 0.990 , respectively)

\section{OARs}

Depending on the margins used, dose distributions of the OARs that did not comply with the planning objectives are shown in Table 4. Only the spinal cord, lens,

Table 4 Comparison of NPC patients not fulfilling dose-volume histogram constraints for plans with pre-correction margins and for plans with post-correction margins

\begin{tabular}{|c|c|c|c|c|c|}
\hline Organ & & Objective & $\begin{array}{l}\text { Plan with pre-correction } \\
\text { margins }[n(\%)]\end{array}$ & $\begin{array}{l}\text { Plan with post-correction } \\
\text { margins }[n(\%)]\end{array}$ & Reduction (\%) \\
\hline \multirow[t]{4}{*}{ Targets } & Any PTV & $V_{93 \%} \geq 99 \%$ & $0(0)$ & $0(0)$ & 0.0 \\
\hline & & $V_{100 \%} \geq 95 \%$ & $0(0)$ & $0(0)$ & 0.0 \\
\hline & PTV_7000 & $V_{77 G y} \leq 20 \%$ & $0(0)$ & $0(0)$ & 0.0 \\
\hline & & $V_{80.5 G y} \leq 5 \%$ & $0(0)$ & $0(0)$ & 0.0 \\
\hline \multirow[t]{23}{*}{ OARs } & BrainStem & $D_{\max } \leq 54 \mathrm{~Gy}$ & $23(57.5)$ & $22(55.0)$ & 4.3 \\
\hline & BrainStem_PRV & $\mathrm{D}_{1 \%} \leq 60 \mathrm{~Gy}$ & $12(30.0)$ & $8(20.0)$ & 33.3 \\
\hline & SpinalCord & $D_{\max } \leq 45 \mathrm{~Gy}$ & $0(0)$ & $0(0)$ & 0.0 \\
\hline & SpinalCord_PRV & $\mathrm{D}_{1 \%} \leq 50 \mathrm{~Gy}$ & $0(0)$ & $0(0)$ & 0.0 \\
\hline & OpticNerve_L & $D_{\max } \leq 50 \mathrm{~Gy}$ & $21(52.5)$ & $19(47.5)$ & 9.5 \\
\hline & OpticNerve_L_PRV & $\mathrm{D}_{1 \%} \leq 54 \mathrm{~Gy}$ & $21(52.5)$ & $17(42.5)$ & 19.0 \\
\hline & OpticNerve_R & $D_{\max } \leq 50 \mathrm{~Gy}$ & $22(55.0)$ & $19(47.5)$ & 13.6 \\
\hline & OpticNerve_R_PRV & $\mathrm{D}_{1 \%} \leq 54 \mathrm{~Gy}$ & $21(52.5)$ & $19(47.5)$ & 9.5 \\
\hline & Chiasm & $\mathrm{D}_{\max } \leq 50 \mathrm{~Gy}$ & $26(65.0)$ & $26(65.0)$ & 0.0 \\
\hline & Chiasm_PRV & $\mathrm{D}_{1 \%} \leq 54 \mathrm{~Gy}$ & $27(67.5)$ & $27(67.5)$ & 0.0 \\
\hline & Lens & $\mathrm{D}_{\max }<25 \mathrm{~Gy}$ & $0(0)$ & $0(0)$ & 0.0 \\
\hline & Parotids & $V_{20 G y}>20 \mathrm{cc}$ (both glands) & $40(100)$ & $40(100)$ & 0.0 \\
\hline & & $\mathrm{D}_{50 \%}<30$ Gy (at least one gland) & $28(70.0)$ & $19(47.5)$ & 32.1 \\
\hline & Parotid_L & $\mathrm{D}_{\text {mean }}<26 \mathrm{~Gy}$ & $40(100)$ & $40(100)$ & 0.0 \\
\hline & Parotid_R & $\mathrm{D}_{\text {mean }}<26 \mathrm{~Gy}$ & $40(100)$ & $40(100)$ & 0.0 \\
\hline & TemporalLobe_L & $D_{\max } \leq 60 \mathrm{~Gy}$ & $39(97.5)$ & $38(95.0)$ & 2.6 \\
\hline & & $\mathrm{D}_{1 \%} \leq 65 \mathrm{~Gy}$ & $15(37.5)$ & $12(30.0)$ & 20.0 \\
\hline & TemporalLobe_R & $D_{\max } \leq 60 \mathrm{~Gy}$ & $39(97.5)$ & $38(95.0)$ & 2.6 \\
\hline & & $\mathrm{D}_{1 \%} \leq 65 \mathrm{~Gy}$ & $16(40.0)$ & $13(32.5)$ & 18.8 \\
\hline & Mandible/TM joint & $D_{1 c c} \leq 75$ Gy & $0(0)$ & $0(0)$ & 0.0 \\
\hline & Ear_Inner_L & $V_{55 G y} \leq 5 \%$ & $30(75.0)$ & $27(67.5)$ & 10.0 \\
\hline & Ear_Inner_R & $V_{55 G y} \leq 5 \%$ & $34(85.0)$ & $26(65.0)$ & 23.5 \\
\hline & Larynx & $\mathrm{D}_{\text {mean }}<45 \mathrm{~Gy}$ & $31(77.5)$ & $28(70.0)$ & 9.7 \\
\hline
\end{tabular}

OAR, organ at risk; PTV, planning target volume; PTV_7000, the PTV of gross tumor volume; PRV, planning risk volume; L, left; R, right; TM, temporomandibular; $\mathrm{V}_{93 \% \text {, }}$ percentage volume receiving at least $93 \%$ of prescribed dose; $V_{100 \%}$, percentage volume receiving at least $100 \%$ of prescribed dose; $V_{77 G y}$, percentage volume receiving at least $77 \mathrm{~Gy} ; \mathrm{V}_{80.5 \mathrm{~Gy}}$, percentage volume receiving at least $80.5 \mathrm{~Gy} ; \mathrm{D}_{1 \%}$, dose received by $1 \%$ of the volume; $\mathrm{D}_{\text {max }}$, maximum dose; $\mathrm{V}_{20 \mathrm{~Gy}}$, volume receiving less than $20 \mathrm{~Gy}$; $c c_{\text {, }}$ cubic centimeter; $D_{50 \%}$, dose received by $50 \%$ of the volume; $D_{\text {mean }}$, mean dose; $D_{1 c c}$, dose received by 1 cc volume; $V_{55 G y}$, percentage volume receiving at least 55 Gy. 
Table 5 Dosimetric comparison of plans with pre-correction margins and plans with post-correction margins for the PTVs in 40 NPC patients

\begin{tabular}{|c|c|c|c|c|c|}
\hline Target & Dose index & Plan with pre-correction margins & Plan with post-correction margins & Difference & $P$ value \\
\hline \multirow[t]{5}{*}{ PTV_7000 } & $\mathrm{D}_{2 \%}(\mathrm{~Gy})$ & $76.2 \pm 0.84$ & $75.97 \pm 0.79$ & $0.28 \pm 0.57$ & 0.003 \\
\hline & $\mathrm{D}_{50 \%}(\mathrm{~Gy})$ & $73.79 \pm 0.43$ & $73.59 \pm 0.38$ & $0.19 \pm 0.32$ & $<0.001$ \\
\hline & $\mathrm{D}_{95 \%}(\mathrm{~Gy})$ & $71.05 \pm 0.32$ & $71.06 \pm 0.26$ & $-0.01 \pm 0.16$ & 0.738 \\
\hline & $\mathrm{D}_{98 \%}(\mathrm{~Gy})$ & $69.53 \pm 1.34$ & $69.80 \pm 1.01$ & $-0.27 \pm 0.44$ & $<0.001$ \\
\hline & $\mathrm{HI}$ & $0.09 \pm 0.03$ & $0.08 \pm 0.02$ & $0.01 \pm 0.01$ & $<0.001$ \\
\hline \multirow[t]{5}{*}{ PTV_6000 } & $D_{2 \%}(G y)$ & $75.77 \pm 0.85$ & $75.44 \pm 0.80$ & $0.33 \pm 0.48$ & $<0.001$ \\
\hline & $\mathrm{D}_{50 \%}(\mathrm{~Gy})$ & $71.18 \pm 1.29$ & $70.79 \pm 1.22$ & $0.39 \pm 0.30$ & $<0.001$ \\
\hline & $\mathrm{D}_{95 \%}(\mathrm{~Gy})$ & $64.00 \pm 1.06$ & $63.85 \pm 1.06$ & $0.16 \pm 0.44$ & 0.030 \\
\hline & $\mathrm{D}_{98 \%}(\mathrm{~Gy})$ & $62.33 \pm 1.04$ & $62.34 \pm 1.07$ & $-0.02 \pm 0.45$ & 0.805 \\
\hline & $\mathrm{HI}$ & $0.19 \pm 0.02$ & $0.18 \pm 0.02$ & $0.01 \pm 0.01$ & 0.010 \\
\hline \multirow[t]{5}{*}{ PTV_5600 } & $D_{2 \%}(G y)$ & $74.60 \pm 1.04$ & $74.33 \pm 1.06$ & $0.27 \pm 0.38$ & $<0.001$ \\
\hline & $\mathrm{D}_{50 \%}(\mathrm{~Gy})$ & $62.58 \pm 0.77$ & $62.41 \pm 0.83$ & $0.17 \pm 0.40$ & 0.009 \\
\hline & $D_{95 \%}(G y)$ & $57.81 \pm 0.56$ & $57.72 \pm 0.54$ & $0.09 \pm 0.22$ & 0.018 \\
\hline & $D_{98 \%}(G y)$ & $55.69 \pm 1.06$ & $55.70 \pm 1.08$ & $-0.01 \pm 0.35$ & 0.990 \\
\hline & $\mathrm{HI}$ & $0.30 \pm 0.03$ & $0.29 \pm 0.03$ & $0.01 \pm 0.01$ & 0.002 \\
\hline
\end{tabular}

All data are presented as mean \pm SD of 40 patients. $\mathrm{Hl}$, homogeneity index; $\mathrm{D}_{2 \%}$, dose received by $2 \%$ of the volume; $\mathrm{D}_{50 \%}$, dose received by $50 \%$ of the volume; $D_{95 \%}$, dose received by $95 \%$ of the volume; $D_{98 \%}$, dose received by $98 \%$ of the volume. Other abbreviations as in Tables 3 and 4 .

Table 6 Dosimetric comparisons of plans with pre-correction margins and plans with post-correction margins for OARs in 40 NPC patients

\begin{tabular}{|c|c|c|c|c|c|}
\hline Organ & Dose index & Plans with pre-correction margins & Plans with post-correction margins & Difference & $P$ value \\
\hline BrainStem_PRV & $D_{1 \%}(G y)$ & $56.93 \pm 5.48$ & $55.62 \pm 5.23$ & $1.31 \pm 1.08$ & $<0.001$ \\
\hline SpinalCord_PRV & $D_{1 \%}(G y)$ & $37.95 \pm 3.81$ & $36.91 \pm 3.45$ & $1.04 \pm 1.41$ & $<0.001$ \\
\hline OpticNerve_L_PRV & $D_{1 \%}(G y)$ & $51.89 \pm 15.01$ & $50.97 \pm 14.48$ & $0.91 \pm 2.20$ & 0.012 \\
\hline OpticNerve_R_PRV & $\mathrm{D}_{1 \%}(\mathrm{~Gy})$ & $51.58 \pm 16.49$ & $50.90 \pm 16.57$ & $0.68 \pm 2.81$ & 0.134 \\
\hline Chiasm_PRV & $\mathrm{D}_{1 \%}(\mathrm{~Gy})$ & $58.84 \pm 13.35$ & $58.63 \pm 12.90$ & $0.21 \pm 2.36$ & 0.578 \\
\hline Lens_L & $D_{\max }(G y)$ & $5.85 \pm 1.81$ & $5.81 \pm 1.82$ & $0.04 \pm 0.23$ & 0.327 \\
\hline Lens_R & $D_{\max }(G y)$ & $5.88 \pm 1.86$ & $5.84 \pm 1.84$ & $0.04 \pm 0.21$ & 0.223 \\
\hline \multirow[t]{2}{*}{ Parotid_L } & $D_{\text {mean }}(G y)$ & $37.31 \pm 4.29$ & $34.66 \pm 4.17$ & $2.65 \pm 1.33$ & $<0.001$ \\
\hline & $V_{30 G y}(\%)$ & $60.81 \pm 15.83$ & $53.35 \pm 15.39$ & $7.46 \pm 7.18$ & $<0.001$ \\
\hline \multirow[t]{2}{*}{ Parotid_R } & $D_{\text {mean }}(G y)$ & $37.31 \pm 4.08$ & $34.44 \pm 4.20$ & $2.87 \pm 1.42$ & $<0.001$ \\
\hline & $\mathrm{V}_{30 \mathrm{~Gy}}(\%)$ & $61.16 \pm 15.25$ & $52.27 \pm 15.92$ & $8.89 \pm 8.51$ & $<0.001$ \\
\hline TemporalLobe_L & $D_{1 \%}(G y)$ & $63.67 \pm 6.94$ & $62.73 \pm 7.47$ & $0.94 \pm 2.17$ & 0.009 \\
\hline TemporalLobe_R & $\mathrm{D}_{1 \%}(\mathrm{~Gy})$ & $64.58 \pm 6.24$ & $63.14 \pm 6.35$ & $1.44 \pm 2.03$ & $<0.001$ \\
\hline Mandible_L & $D_{1 \subset c}(G y)$ & $60.24 \pm 3.43$ & $58.56 \pm 4.36$ & $1.67 \pm 2.10$ & $<0.001$ \\
\hline Mandible_R & $D_{1 \subset c}(G y)$ & $60.28 \pm 4.56$ & $58.28 \pm 5.18$ & $2.00 \pm 1.83$ & $<0.001$ \\
\hline TMjoint_L & $D_{1 c c}(G y)$ & $44.67 \pm 10.59$ & $42.16 \pm 10.79$ & $2.51 \pm 4.24$ & 0.001 \\
\hline TMjoint_R & $D_{1 c c}(G y)$ & $45.46 \pm 12.14$ & $43.40 \pm 10.99$ & $2.06 \pm 3.25$ & $<0.001$ \\
\hline Ear_Inner_L & $D_{\text {mean }}(G y)$ & $49.36 \pm 7.98$ & $47.69 \pm 7.67$ & $1.67 \pm 1.61$ & $<0.001$ \\
\hline Ear_Inner_R & $D_{\text {mean }}(G y)$ & $49.86 \pm 8.42$ & $48.00 \pm 8.17$ & $1.87 \pm 2.22$ & $<0.001$ \\
\hline Larynx & $D_{\text {mean }}(G y)$ & $47.09 \pm 2.57$ & $45.98 \pm 2.52$ & $1.11 \pm 1.46$ & $<0.001$ \\
\hline
\end{tabular}

All data are presented as mean \pm SD of 40 patients. $V_{30 G y}$, percentage volume receiving at least 30 Gy. Other abbreviations as in Tables 3 and 4. 
mandible, and temporomandibular (TM) joint dose distributions complied with the planning objectives for all patients, irrespective of the margin strategy. However, with margin reductions, there was a $33.3 \%$ reduction in the number of patients who did not fulfill the criteria of $\mathrm{D}_{1 \%}<60$ Gy for BrainStem_PRV and a 32.1\% reduction in the planning objective of $D_{50 \%}<30$ Gy for the parotids. Dose distribution improvements from margin reductions for the brainstem and the parotids were consistently better than those for other OARs.

A favorable dosimetric impact of margin reductions on OARs was observed (Table 6). The average DVH for all the OARs, comparing the two different margin strategies for the entire patient cohort, is shown in Figure 1. Significant differences in dosimetric parameters between the two margin strategies were observed for all OARs

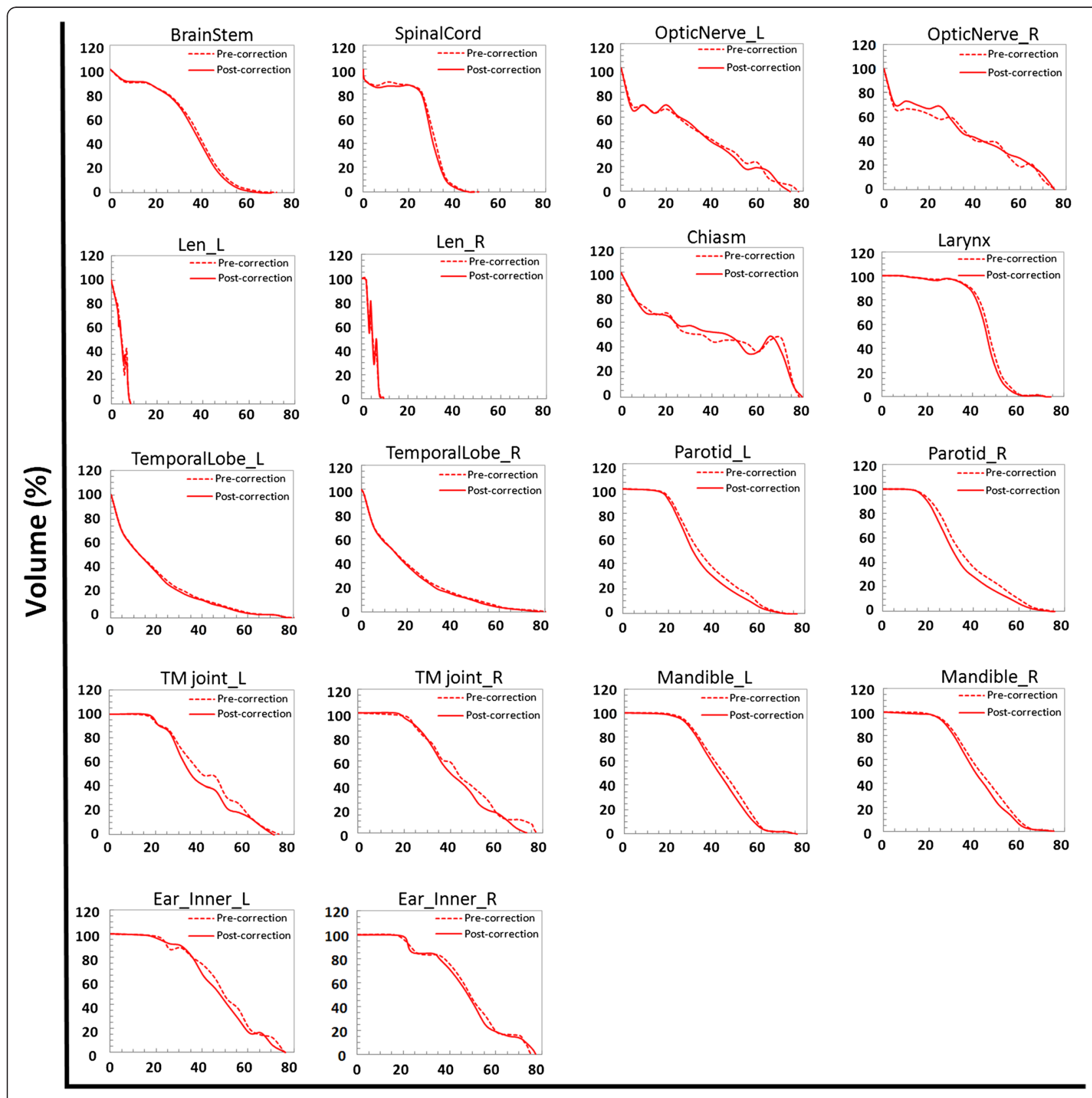

Dose (Gy)

Figure 1 Average dose volume histograms of plans with pre-correction margins and plans with post-correction margins for all 40 patients with nasopharyngeal carcinoma. Each picture is based on the average dose volume histogram to an organ at risk. L, left; R, right; TM, temporomandibular. 
$(P<0.05)$, apart from the PRV of the right optic nerve (OpticNerve_R_PRV), the PRV of the chiasm (Chaism PRV), and the PRV of the lens. With margin reductions, the maximum doses to BrainStem_PRV, SpinalCord_PRV, and the PRV of the left optic nerve (OpticNerve_L_PRV) were decreased by $2.3 \%, 2.6 \%$, and $1.7 \%$, respectively, whereas the mean doses to the left parotid, right parotid, left inner ear, right inner ear, and larynx were decreased by $7.1 \%, 7.7 \%, 3.3 \%, 3.6 \%$, and $2.3 \%$, respectively. Additionally, the $\mathrm{D}_{1 \%}$ decreased at a rate of $1.5 \%$ and $2.2 \%$ in the left and right temporal lobes, respectively, and the dose received by 1 cubic centimeter volume $\left(D_{1 \text { cc }}\right)$ reduced by $2.8 \%, 3.4 \%$, $5.4 \%$, and $3.7 \%$ in the left mandible, right mandible, left TM joint, and right TM joint, respectively. Sparing of the left and right parotids was consistently better than that observed with other OARs. Planning objectives for healthy tissue were not formalized numerically, but the strategy was to minimize the involvement of these tissues.

Our results showed that the DVH parameters for each OAR didn't decrease significantly with margin reduction when dividing the patients into different groups according to the tumor size $(P>0.05)$. To analyze the association between the DVH parameters and T category, we divided the patients into 4 groups according to the T category. Our results showed that, only the percentage volume receiving at least $60 \mathrm{~Gy}\left(\mathrm{~V}_{60 \mathrm{~Gy}}\right)$ of the brainstem significantly decreased with shrank margin in patients with T4 lesions $(P=0.001)$. However, the reductions in the maximum doses to the brainstem, optic nerve, chiasm, and right lens, the mean doses to the left parotid and ear, and $\mathrm{D}_{1 \mathrm{cc}}$ to the right TM joint were more apparent in patients with T4 lesions, but no significant difference was observed among the 4 groups.

\section{Discussion}

Many reports have demonstrated that the dose distribution can be improved by minimizing PTV margin [6-8]. According to our knowledge, this study is the first to report the dosimetric effect of PTV margin reduction during the treatment of NPC patients and demonstrate improvements both in OAR sparing and target coverage.

Recent studies have described PTV margin reductions for multiple correction protocols in radiotherapy for head and neck cancer. Den et al. [11] and Wang et al. [12] reported that CBCT online correction with 2-mm tolerance resulted in $40 \%-60 \%$ and $51 \%-68 \%$ reduction in PTV margins, respectively. Velec et al. [15] concluded that CBCT facilitated a $13 \%-39 \%$ reduction in PTV margins with a combination of online correction with 3-mm tolerance and offline correction. Rates of margin reductions in our study are similar to those reported by Den et al. [11] and Wang et al. [12] but greater than those reported by Velec et al. [15]. This difference may be due to the various correction thresholds and correction protocols used in these studies. Generally, a smaller correction threshold was associated with a higher rate of margin reductions. Additionally, online correction can reduce both systematic error and random error, whereas offline correction can only reduce systematic error $[16,17]$. Therefore, the rate of margin reduction can be increased with online correction.

Improvements in dose distributions with margin reductions have been reported for many tumors, but not for NPC. Grills et al. [7] reduced the margins surrounding lung tumors from $9-13 \mathrm{~mm}$ to $2-4 \mathrm{~mm}$ with online correction using CBCT, and this reduction in margins resulted in $23 \%-32 \%$ dose reduction in average lung dose, $19 \%-27 \%$ dose reduction in maximum spinal cord dose, and $10 \%-22 \%$ dose reduction in maximum esophageal dose. In our study, the reduced margins decreased the dose to OARs with values ranging from $1.5 \%$ to $7.7 \%$ and provided better target coverage. Compared with the result reported for lung cancer, the margin reduction for NPC was smaller, and the decreases of OAR doses caused by margin reduction were less. This finding may have resulted from higher reproducibility and stability of the patient position for NPC than those for thoracic tumors. Our results demonstrate the dosimetric advantage of reduced margins using CBCT online correction for NPC.

Additionally, in our study, planning objectives of the brainstem and parotids were not fulfilled in $57.5 \%$ and $70.0 \%$ of patients, respectively, for plans with precorrection margins. However, among those patients who did not have a $\mathrm{D}_{1 \%}$ less than 60 Gy for BrainStem_PRV, a $33.3 \%$ margin reduction was observed. Additionally, there was a $32.1 \%$ reduction in patients who did not fulfill the planning objective of $\mathrm{D}_{50 \%}<30$ Gy for the parotids. The pass rates of the radiotherapy plan from margin reductions for the brainstem and parotids were consistently higher than those observed for other OARs. This observation may be explained by the close proximity of the tumors to both the brainstem and parotids, and margin reductions result in a decrease in the overlap volume between the two OARs and the tumors, thereby decreasing the volume of the OARs irradiated at high dose levels. Therefore, margin reductions can achieve the planning objectives of the OARs, thereby improving the acceptance and tolerance rates of the radiotherapy plan.

Sparing of the parotid glands was most obvious, with a decrease in the mean dose up to $7.7 \%$. This result suggests that patients could have a dose decrease if planned with small PTV margins and treated with the post-correction position, thereby enhancing treatment efficacy. Additionally, with $\mathrm{CBCT}$ online correction, the margins decreased to $2.5 \mathrm{~mm}, 1.4 \mathrm{~mm}$, and $1.5 \mathrm{~mm}$ in the ML, SI, and AP directions, respectively. This reduction resulted in a decrease in mean doses of 2.65 Gy and 2.87 Gy to the left and right parotids, respectively. These results are similar to those 
reported by van Asselen et al. [18], who observed that the mean dose to the parotids increases linearly with increasing margins by approximately $1.3 \mathrm{~Gy} / \mathrm{mm}$. The occurrence of radiation adverse effects, which affect the quality of life, is related to the radiation dose. However, the present study involved only dosimetry. Further research is warranted to confirm whether the decrease of OAR dose observed in our study may reduce the incidence of adverse effects.

Our study showed that only the $\mathrm{V}_{60 \mathrm{~Gy}}$ of the brainstem significantly decreased with shrank margin in patients with T4 lesions compared with those with T1-3 lesions $(P<0.01)$. This finding indicates that the high dose region of the brainstem was reduced significantly for patients with T4 lesions compared with those with other stage diseases. As we know, the value of $\mathrm{V}_{60 \mathrm{~Gy}}$ is rarely greater than 0 in patients with $\mathrm{T} 1$ lesions, whereas it is rarely greater than $10 \%$ for patients with T4 lesions; it is larger for patients with $\mathrm{T} 4$ lesions than that for those with T1-3 lesions. Therefore, if there is a small reduction in PTV margins, the reduction of $\mathrm{V}_{60 \mathrm{~Gy}}$ is more apparent for patients with T4 lesions than for patients with T1-3 lesions.

The reductions in the maximum dose to the brainstem, optic nerve, chiasm, and right lens, in the mean dose to the left parotid and ear, and in $\mathrm{D}_{1 \mathrm{cc}}$ to the right TM joint were more apparent for patients with T4 lesions compared with other groups of patients, but no significant difference was observed. A possible reason for this result was that the reductions in PTV margins were not large enough to achieve statistical significance among these groups. Another reason was the limited data used for the analysis: our study included only 40 patients, with 7-15 patients in each group.

Because many critical normal structures are in close proximity to the nasopharynx, it is important to protect OARs without compromising PTV coverage in NPC. First, accurate and consistent OAR delineation in NPC is critical for organ protection. Inaccurate delineation will mislead treatment planning, resulting in OAR overdose or inadequate target volume coverage. Sun et al. [19] found that different contouring methods can make the volume and dosimetric parameters of organs significantly different. Second, shrunken targets contributed to the protection of OARs. The common methods of narrowing the targets are reducing the PTV margins by improving the placement accuracy or modifying the target during the course of therapy. Furthermore, the sufficient sparing of critical normal structures in NPC patients could be achieved by the use of IMRT. As we know, IMRT offers superior dose conformity to tumor targets with relative sparing of critical organs. Results from retrospective and prospective studies have confirmed the efficacy of IMRT on disease control as well as the benefit in OAR sparing. Finally, appropriate dose limit parameters to OARs also contribute to sparing of critical structures. There are various ways to protect the OARs, and the most suitable method to decrease the radiation dose to OARs should be adopted.

\section{Conclusion}

This study is the first to quantify the magnitude of sparing of OARs due to margin reductions with $\mathrm{CBCT}$ online correction in IMRT for the management of NPC. Our results confirmed improvements both in OAR sparing and target coverage, and the parotid gland benefited most from the online imaging-guided approach. However, prospective randomized trials should be performed to confirm whether the dosimetric benefit observed in our study can be translated into clinical benefit.

\section{Competing interests}

The authors declare that they have no competing interests.

\section{Authors' contributions}

The authors contributions are the following: YPM, WJY, and JM contributed to literature research, study design, interpretation of findings, and writing of the manuscript. FC, JLF, and ZYQ contributed to data collection. RG, GSZ, and MZL contributed to data analysis. YS contributed to critical review of data analyses, interpretation of findings, and critical revising of the manuscript. All authors read, revised, and approved the final manuscript.

\section{Acknowledgements}

This work was supported by grants from the Health \& Medical Collaborative Innovation Project of Guangzhou City, China (No. 201400000001), the Planned Science and Technology Project of Guangdong Province (No. 2012B031800092), the Medical Science Foundation of Guangdong Province (No. B2012135), and the Cultivating Foundation of Education Bureau of Guangdong Province (No. LYM11001).

\section{Author details}

${ }^{1}$ Department of Radiation Oncology, Sun Yat-sen University Cancer Center; State Key Laboratory of Oncology in South China; Collaborative Innovation Center for Cancer Medicine, Guangzhou, Guangdong 510060, Peoples Republic of China. 2Department of Radiation Oncology, Cancer Center of Guangzhou Medical University, Guangzhou, Guangdong 510095, Peoples Republic of China.

Received: 25 August 2014 Accepted: 9 February 2015

Published online: 20 May 2015

\section{References}

1. Chan AT, Teo PM, Johnson PJ. Nasopharyngeal carcinoma. Ann Oncol. 2002;13:1007-15

2. Kam MK, Teo PM, Chau RM, Cheung KY, Choi PH, Kwan WH, et al. Treatment of nasopharyngeal carcinoma with intensity-modulated radiotherapy: the Hong Kong experience. Int J Radiat Oncol Biol Phys. 2004;60:1440-50.

3. Lin S, Pan J, Han L, Zhang X, Liao X, Lu JJ. Nasopharyngeal carcinoma treated with reduced-volume intensity-modulated radiation therapy: report on the 3-year outcome of a prospective series. Int J Radiat Oncol Biol Phys. 2009;75:1071-8.

4. Kam MK, Leung SF, Zee B, Chau RM, Suen JJ, Mo F, et al. Prospective randomized study of intensity-modulated radiotherapy on salivary gland function in early-stage nasopharyngeal carcinoma patients. J Clin Oncol. 2007;25:4873-9.

5. Petsuksiri J, Sermsree A, Thephamongkhol K, Keskool P, Thongyai K, Chansilpa $Y$, et al. Sensorineural hearing loss after concurrent chemoradiotherapy in nasopharyngeal cancer patients. Radiat Oncol. $2011 ; 6: 19$

6. Hanley J, Debois MM, Mah D, Mageras GS, Raben A, Rosenzweig K, et al. Deep inspiration breath-hold technique for lung tumors: the potential value of target immobilization and reduced lung density in dose escalation. Int J Radiat Oncol Biol Phys. 1999;45:603-11.

7. Grills IS, Hugo G, Kestin LL, Galerani AP, Chao KK, Wloch J, et al. Image-guided radiotherapy via daily online cone-beam $C T$ substantially reduces margin 
requirements for stereotactic lung radiotherapy. Int J Radiat Oncol Biol Phys. 2008;70:1045-56.

8. Song S, Yenice KM, Kopec M, Liauw SL. Image-guided radiotherapy using surgical clips as fiducial markers after prostatectomy: a report of total setup error, required PTV expansion, and dosimetric implications. Radiother Oncol. 2012;103:270-4

9. Stutzel J, Oelfke U, Nill S. A quantitative image quality comparison of four different image guided radiotherapy devices. Radiother Oncol. 2008;86:20-4.

10. Korreman S, Rasch C, McNair H, Verellen D, Oelfke U, Maingon P, et al. The European Society of Therapeutic Radiology and Oncology-European Institute of Radiotherapy (ESTRO-EIR) report on 3D CT-based in-room image guidance systems: a practical and technical review and guide. Radiother Oncol. 2010;94:129-44.

11. Den RB, Doemer A, Kubicek G, Bednarz G, Galvin JM, Keane WM, et al. Daily image guidance with cone-beam computed tomography for head-and neck cancer intensity-modulated radiotherapy: a prospective study. Int J Radiat Oncol Biol Phys. 2010;76:1353-9.

12. Wang J, Bai S, Chen N, Xu F, Jiang X, Li Y, et al. The clinical feasibility and effect of online cone beam computer tomography-guided intensity-modulated radiotherapy for nasopharyngeal cancer. Radiother Oncol. 2009;90:221-7.

13. van Herk M, Remeijer P, Rasch C, Lebesque JV. The probability of correct target dosage: dose-population histograms for deriving treatment margins in radiotherapy. Int J Radiat Oncol Biol Phys. 2000;47:1121-35.

14. Burrows J. Use of Monte Carlo simulations in the assessment of calibration strategies-Part I: an introduction to Monte Carlo mathematics. Bioanalysis. 2013:5:935-43.

15. Velec M, Waldron JN, O'Sullivan B, Bayley A, Cummings B, Kim JJ, et al. Cone-beam CT assessment of interfraction and intrafraction setup error of two head-and-neck cancer thermoplastic masks. Int J Radiat Oncol Biol Phys. 2010;76:949-55.

16. Zeidan OA, Langen KM, Meeks SL, Manon RR, Wagner TH, Willoughby TR, et al. Evalution of image-guidance protocols in the treatment of head and neck cancers. Int J Radiat Oncol Biol Phys. 2007;67:670-7.

17. Litzenberg DW, Balter JM, Lam KL, Sandler HM, Ten Haken RK. Retrospective analysis of prostate cancer patients with implanted gold markers using off-line and adaptive therapy protocols. Int J Radiat Oncol Biol Phys. 2005;63:123-33.

18. van Asselen B, Dehnad H, Raaijmakers CP, Roesink JM, Lagendijk JJ, Terhaard CH. The dose to the parotid glands with IMRT for oropharyngeal tumor: the effect of reduction of positioning margins. Radiother Oncol. 2002;64:197-204.

19. Sun $Y, Y u X L$, Luo W, Lee AW, Wee JT, Lee N, et al. Recommendation for a contouring method and atlas of organs at risk in nasopharyngeal carcinoma patients receiving intensity-modulated radiotherapy. Radiother Oncol. 2014;110:390-7.

\section{Submit your next manuscript to BioMed Central and take full advantage of:}

- Convenient online submission

- Thorough peer review

- No space constraints or color figure charges

- Immediate publication on acceptance

- Inclusion in PubMed, CAS, Scopus and Google Scholar

- Research which is freely available for redistribution

Submit your manuscript at www.biomedcentral.com/submit 